\title{
Für eine Aufwertung der kommunalen Jugendhilfe
}

\section{Vorschläge für eine zeitgemäße Finanzierung und Struktur}

\author{
Wolfgang Hinte, Oswald Menninger und Georg Zinner
}

The children and youth welfare law offered several options for professional renewal. However, it does not offer incentives for synergy when looking at the gateway of youth welfare. The current structures of financing are not geared to strengthen the educational task of regular systems of provision for families, child care services, leisure time services for children and youth and schools. A flat rate system, defining the budget for youth welfare could open rigid structures.

D'une part, la loi sur la jeunesse et l'enfance ont apporté différentes solutions de renouveau professionnel. Cependant, quand on regarde la voie de l'aide à la jeunesse, on remarque qu'elle ne propose pas de mesures incitatives aux synergies. En particulier, les structures actuelles de financement ne sont pas conçues pour renforcer le rôle pédagogique des systèmes traditionnels d'aide aux familles, d'aide à l'enfance, des services récréationnels pour les enfants et les jeunes et des écoles. Un système forfaitaire, définissant le budget d'aide à la jeunesse, pourrait permettre d'ouvrir les structures rigides.

\section{Prof. Dr. Wolfgang Hinte ist}

geschäftsführender Leiter des Instituts für Stadtteilbezogene Soziale Arbeit und Beratung (ISSAB), eine wissenschaftliche Einrichtung der Universität DuisburgEssen.

Oswald Menninger ist Geschäftsführer des Paritätischen Wohlfahrtsverbandes Landesverband Berlin e. V.

Georg Zinner ist Geschäftsführer des

Nachbarschaftsheims Schöneberg e.V. in

Berlin. Der Verein ist Träger von

Einrichtungen der Kinder- und

Jugendhilfe und der ambulanten und stationären Pflege.

Die Autoren danken Johannes Groppe und Detlef Pawollek für Anregungen zu diesem Beitrag.

E-Mail issab@uni-essen.de
Das Kinder- und Jugendhilfegesetz hat einerseits manche fachliche Innovation ermöglicht, bietet aber andererseits kaum Anreize für Synergien an den offenkundigen Schnittstellen der Jugendhilfe. Insbesondere die jetzigen Finanzierungsstrukturen sind nicht darauf ausgerichtet, die Regelsysteme Familie, Kita, Kinder- und Jugendfreizeiteinrichtungen und Schule bei ihrem Sozialisationsauftrag zu stärken. Ein festgeschriebenes kommunales Budget für Jugendhilfe könnte erstarrte Strukturen aufbrechen helfen.

\section{1.}

Das Kinder- und Jugendhilfegesetz (SGB VIII) war zum Zeitpunkt seiner Einführung im Jahre 1991 ein überfälliges Gesetz, das die weitgehend auf staatliche Kontrolle und Ordnung zielenden Vorgängergesetze ablöste.

Insbesondere die Unterstützung von Kindern, Jugendlichen und ihren Familien in akuten und andauernden Krisensituationen hat sich durch das neue Gesetz grundlegend geändert. Bis weit in die 1980er Jahre hinein beschränkten sich die Unterstützungsmöglichkeiten für Kinder und Jugendliche in Krisensituationen weitgehend auf Fremdplatzierungen, die entweder gegen den Willen der Eltern angeordnet oder auf Antrag der Eltern nach oft undurchsichtigen Entscheidungen mit großzügiger Geste durch den öffentlichen Träger gewährt wurden.

Bezugspunkte für die professionelle Arbeit in den stationären Einrichtungen waren tendenziell eher der Strafvollzug und die preußische Kasernenhofpädagogik als fortschrittliche Konzepte der Heimpädagogik. In diesem Bereich der Jugendhilfe hat das Kinder- und Jugendhilfegesetz die Entwicklung und die praktische Umsetzung fortschrittlicher, auf Beteiligung gerichteter sozialpädagogischer Konzepte unterstützt. Die Entwicklung und Umsetzung professioneller Handlungskonzepte in diesen Bereichen der Jugendhilfe in den zurückliegenden 15 Jahren hat sich positiv auf die Verbesserung von Lebensbedingungen insbesondere von benachteiligten Kindern und Jugendlichen ausgewirkt.

Bei aller Kritik an verschiedenen Entwicklungen, die im Rahmen der jüngsten Reformbemühungen (insbesondere bei der Umsetzung des Fachkonzepts "Sozialraumorientierung «) in der Jugendhilfe immer wieder laut werden, gibt es in der Fachöffentlichkeit einen breiten Grundkonsens darüber, dass das Kinder- und Jugendhilfegesetz eine unverzichtbare gesetzliche Grundlage für Bestand und Weiterentwicklung einer fachlich hochwertigen Kinder- und Jugendhilfe ist.

\section{2.}

In letzter Zeit häuft sich die Kritik sowohl am Kinder- und Jugendhilfegesetz als auch an der Art der Umsetzung auf kommunaler Ebene. Kritisch gesehen werden die »Familienlastigkeit « des Gesetzes, die fehlende Verankerung von Kinderrechten, die versäult aufgezählten Hilfen sowie die durch das Gesetz beförderte Tendenz, auf kommunaler Ebene durch gesetzlich garantierte Unterstützungsleistungen den Regelsystemen zu ermöglichen, schwierige oder unliebsame Kinder und Jugendliche auszusondern.

In der Tat bietet das Kinder- und Jugendhilfegesetz in der aktuellen Form keinerlei Grundlagen und Anreize, Regeleinrichtungen im Bereich der Kinder- und Jugendhilfe ausreichend gut auszustatten. Vielmehr werden die Geldströme relativ einseitig in den Bereich der konstatierten sozialen Auffälligkeit (»Leistungsberechtigung «) geleitet, in dem dann häufig sehr mühsam bezogen auf den attestierten Einzelfall solche Strukturen (etwa durch zahlreiche Träger der Erziehungshilfen) aufgebaut werden müssen, in denen verspätet ähnliche Arbeit geleistet wird, die in weiten Teilen der Arbeit in einer guten 
Regeleinrichtung entspricht. Die Folgen sind u. a.:

- zergliederte und bürokratieaufwendige Finanzierungsstrukturen

- Doppelfinanzierung von einerseits Regelstruktur und andererseits " $\mathrm{Zu}$ sammenbruchs-Struktur «

- Schwächung der Verantwortung der Regelstruktur und vermehrter (unnötig hoher) Einsatz im Bereich des konstatierten »sozialen Bedarfs «

- Aufbau von Doppelstrukturen (etwa durch Tagesgruppen, die teilweise die gleiche Arbeit leisten wie so manche Regeleinrichtung)

- keine Anreize für Synergien an den offenkundigen Schnittstellen der Systeme. So wird etwa die Hausaufgabenhilfe (wenn überhaupt) erst dann aufgebaut, wenn genügend »Fälle« identifiziert wurden; die »soziale Gruppenarbeit « wird erst dann finanziert, wenn eine ausreichende Zahl an Leistungsberechtigten vorhanden ist; der Babysitterdienst wird gar nicht erst finanziert, weil er nicht im Katalog der Hilfen nach $\$ 27 \mathrm{ff}$. SGB VIII auftaucht; das Anti-Aggressions-Training kann allenfalls über Umwege finanziert werden, und dann auch in der Regel nur für die »besonders harten Fälle«.

Das Gerede von Prävention wird Lügen dadurch gestraft, dass die kommunalen Gebietskörperschaften das für die Jugendhilfe im Budget befindliche Geld vornehmlich dafür einsetzen, zum Zeitpunkt des konstatierten Leistungsanspruchs die vorgeschriebene Leistung zu erbringen und selbst diese ist vielerorts nicht mehr garantiert. Und angesichts quantitativ und qualitativ wachsender Leistungsansprüche werden Kürzungen (insbesondere auf dem Hintergrund kurzfristig orientierter Sparpolitik) in den Bereichen vorgenommen, die nicht zum Pflichtkatalog gehören und deren auf den Leistungsbereich wirkende präventive Kraft allenfalls nach einigen Jahren deutlich würde.

Damit wird die ohnehin in Deutschland mangelhafte Infrastruktur der Kinder-, Jugend- und Familienförderung von der Kindertagesstätte über den Hort bis hin zu Kinder- und Jugendfreizeiteinrichtungen und Stadtteileinrichtungen weiter geschwächt, statt - den gesellschaftlichen Erfordernissen entsprechend - ausgebaut.

Solange in der Jugendhilfe fachbereichsspezifische Steuerungsparameter und Trägerkonkurrenz (verbunden mit Überlebenskämpfen) dominieren, wird es nicht gelingen, die quantitativ beachtlichen und qualitativ weiter fortentwickelten Ressourcen der Jugendhilfe gezielt einzusetzen. Derzeit sind insbesondere die Hilfen zur Erziehung eine äußerst kostenintensive und völlig überbürokratisierte "Alternative « zu einer bedarfsorientierten Verbesserung der Regelangebote, insbesondere für benachteiligte Familien (insbesondere alleinerziehende Eltern). Somit geht es nicht um eine sinnvollere Gestaltung der Schnittstelle der Hilfen zur Erziehung zu verschiedenen Regelangeboten oder um eine bessere Kooperation verschiedener Jugendhilfeträger sondern um eine - auch und gerade finanzierungstechnisch abgebildete - Integration der vorhandenen Jugendhilfeangebote.

In diesem Zusammenhang müssen auch die Trägerstrukturen in der Jugendhilfe überprüft und weiterentwickelt werden. Die lange als zentrale Errungenschaft gefeierte Trägervielfalt hat nicht dazu geführt, dass passgenaue Hilfen entwickelt oder durch frühzeitige Unterstützung oder präventive Aktivitäten aufwendige Maßnahmen verhindert werden. Vielmehr ist eine durchaus facettenreiche, vielerorts aber auch undurchsichtige Trägerlandschaft entstanden, in der die Träger auf "Fälle« angewiesen sind und sich - weil sie keine Alternativen haben - "Märkte « erobern und untereinander konkurrieren müssen. Angesichts hoher Planungsunsicherheit sind die Träger genötigt, wirtschaftliche Aspekte in einer Art und Weise zu gewichten, die ansonsten im sozialarbeiterischen Diskurs so gerne beklagt wird. Finanzierungstechnisch geförderte Kooperation und eine engere Anbindung, vielleicht sogar die Integration, von Hilfeanbietern an Stadtteileinrichtungen, Schulen, Kitas oder Jugendzentren würde eine bessere Nutzung von Ressourcen ermöglichen und die Integrationsmöglichkeiten der Regelsysteme erweitern.

\section{3.}

Die Programmatik des »aktivierenden Sozialstaats « wird geradezu ins Gegenteil verkehrt, wenn ein Leistungsgesetz einseitig konzentriert ist auf die Erfüllung von Pflichtansprüchen (allenfalls unter »Mitwirkung « der Beteiligten).

Der gesamte Bereich der systematischen, möglichst rechtlich gesicherten Unterstützung etwa von Selbsthilfe, Bür- gerengagement, Stadtteilarbeit usw., der eine wesentliche Rolle sowohl bei der Verhinderung oder Reduzierung von Leistungsansprüchen wie auch bei der niveauvollen Erfüllung von Leistungsansprüchen spielen könnte, ist entweder gar nicht oder chronisch unterfinanziert. Dies fördert das Delegationsprinzip der Problemlösung an Hilfeinstitutionen statt bürgerschaftliche Problemlösungskompetenz zu fördern und auch zu erwarten. »Familienunterstützende Angebote « etwa sind in aller Munde und programmatisch unstrittig, aber es gibt - außer einigen befristeten Programmen - keinerlei Anreize für eine strategisch agierende Gebietskörperschaft, derlei Angebote in einer Art und Weise auszubauen, dass sie (zumindest mit entsprechender zeitlicher Verzögerung) Wirkungen auf den Leistungsbereich haben oder damit systematisch verschränkt werden. So ist zu erwarten, dass die Initiative der Bundesregierung für "Mehrgenerationenhäuser « nur dann über die Programmlaufzeit hinaus Folgen haben wird, wenn es rechtliche Grundlagen gibt, die die kommunalen Gebietskörperschaften darauf orientieren, derlei Einrichtungen weiter zu finanzieren und sie als einen festen Bestandteil der kommunalen Infrastruktur zu betrachten.

Bei einer Neuausrichtung der Jugendhilfe muss insbesondere die Finanzierung der Jugendhilfe auf den Prüfstand. Ausgehend von der Binsenweisheit, dass die Finanzierungssystematik fachpolitische Handlungsprinzipien einerseits unterstützen und fördern, andererseits im Extremfall konterkarieren kann, muss in eine andere Richtung gedacht werden. Die jetzigen Finanzierungsstrukturen sind nicht danach ausgerichtet, die Regelsysteme Familie, Kita, Kinder- und Jugendfreizeiteinrichtungen und Schule bei ihrem Sozialisationsauftrag zu stärken. Trotz der Finanzierungsnormen im Kinder- und Jugendhilfegesetz wurden die präventiven, niedrigschwelligen Angebote auf Grund der kommunalen Konsolidierungspolitik überall zurückgefahren, weil sie nur sinnvoll durch Zuwendungen finanziert werden können. Zuwendungen sind aber haushaltsrechtlich als »freiwillige Leistungen « definiert, und beim Zwang zur Haushaltskonsolidierung geraten diese politischen »Ermessensleistungen « zuerst unter die Räder - frei nach dem Motto des Berliner Finanzsenators: »Wer pleite ist, hat kein Ermessen mehr. « Das Land Berlin hat 
auch gezeigt, dass die viel beschworenen Rechtsansprüche bei den Hilfen zur Erziehung nicht sakrosankt sind, denn deren Budget wurde in einem Zeitraum von fünf Jahren um fast 40 Prozent abgesenkt. Nennenswerte Rechtsverletzungen waren trotz gesetzlicher Normierungen entgegen allen Erwartungen nicht nachzuweisen. Abgesehen von diesen ohnehin schwierigen Bedingungen sollte die Jugendhilfe nicht abwarten, bis der nächste Ländervorstoß kommt, der die Finanzierung der Jugendhilfeleistungen unter einen gesetzlichen Finanzierungsvorbehalt stellen will, sondern stattdessen eine mutige und ideenreiche $\mathrm{Fi}$ nanzierungsdiskussion führen.

Die Leitplanken dieser Diskussion sollten von der Strategie bestimmt sein, die Kommunen zu zwingen, eine bestimmte Summe zweckgebunden ausschließlich für die Kinder- und Jugendhilfe einzusetzen. Die Berechnungsgröße dieser Summe müsste sich an klassischen Belastungsfaktoren (Anzahl Kinder und Jugendliche, Alleinerziehende, Jugendliche mit Migrationshintergrund, Arbeitslosenquote) ausrichten, die zu einem Jugendeinwohnerwert verdichtet werden (damit es nicht zu einem von der jeweiligen Haushaltslage bestimmten »Ortsrecht « kommt). Die Kommune ist dann frei, diese Mittel sozialräumlich und infrastrukturell einzusetzen. Skeptiker werden einwenden, dass dann das Geld nur noch für »Musikschulen und Reitunterricht für die Kinder der Besserverdienenden « eingesetzt werde. Diesem berechtigten Einwurf kann begegnet werden, indem die Errungenschaft des Kinderund Jugendhilfegesetzes »Leistungsanspruch des Einzelnen « eingebunden bleibt. Ebenfalls gegen eine Fehlverwendung der Mittel könnte das verbindliche Vorschreiben eines kommunalen Jugendförder- und - entwicklungsplanes wirken. Ein solcher Plan evoziert politische Diskussionen über die Jugendhilfe vor Ort und ermuntert die Jugendhilfeakteure, um die beste Verwendung der Mittel produktiv zu streiten. Dabei muss klar sein, dass das Kinder- und Jugendhilfegesetz ein Gesetz für alle Kinder, Jugendlichen und Familien ist und dass jedes Kind und jeder Jugendliche das Recht haben muss, eine attraktive und interessante Palette etwa von Kinderfreizeit- und Jugendförderangeboten in Wohnortnähe nutzen zu können. (Die gegenwärtige Praxis hat dagegen faktisch ein reines Hilfegesetz geschaffen, ähnlich dem SGB II und SGB XII.)
Ein Benchmarking der besten kommunalen Jugendhilfepolitik wäre die logische Folge eines derartigen Finanzierungsansatzes. Eine schlechte kommunale Jugendhilfepolitik ist dann erstens erkennbar und zweitens kann sie sich nicht exkulpieren mit dem Hinweis auf das fehlende Geld, denn vergleichbare Kommunen setzen die gleichen Ressourcen ein.

Unter derartigen Bedingungen kann es zu systematischen Verbesserungen kommen, denn die Interessen aller Akteure werden nicht auf die Sicherung, sondern auf die beste Verwendung der Mittel gelenkt. In diesen Kontext gehören auch Überlegungen, das Kindergeld als bundessteuerfinanzierte Leistung über die örtlichen Jugendämter auszuzahlen. Bei Eltern, die ihre Erziehungspflichten grob
Fachlich ist es heute unstrittig, dass etwa der verstärkte Einsatz von Schulsozialarbeit oder der Ausbau von Stadtteilläden, Nachbarschaftsheimen und Bürgerhäusern massive Beiträge für sinnvolle Prävention und für die Unterstützung von Familien in prekären Situationen leisten könnte. Kaum eine Kommune ist jedoch bereit, dort systematisch zu investieren, sondern man wartet solange, bis der Katastrophenfall eingetreten ist, der dann viel Geld kostet, das man dann zähneknirschend deshalb bezahlt, weil man es bezahlen muss (»Leistungsanspruch «).

Die kurzfristige politische Logik frönt einem Reaktionsmuster, das erst wartet, bis die Notlage eingetreten ist, und dann unter dem Druck der Verhältnisse oder

\section{»Fachbereichsdenken und Träger- konkurrenz verhindern einen optimalen Einsatz der Jugendhilfe- Ressourcen"}

verletzen, sollte die Geldleistung in eine Sachleistung umgewandelt werden können. Sachleistungen sind sozialrechtlich üblich und könnten an eine Elternbildungsmaßnahme gekoppelt werden, bevor die Geldleistung entzogen wird. Das örtliche Jugendamt hätte damit bessere und differenzierte Steuerungsmöglichkeiten, um auf Eltern einzuwirken. Und scheitert dies, stehen die staatlichen Mittel für Leistungen zugunsten der Kinder zur Verfügung. Vor Ort kann dann entschieden werden, ob man Kinder in Heimen unterbringen, die Frühförderung ausbauen, Schwangere beraten oder eher Sozialraumbudgets entwickeln will. Dabei braucht es gesetzliche Minimalregelungen, etwa für den Kinderschutz (der ohnehin durch ein anderes Gesetz geregelt ist) sowie für Hilfen zur Erziehung (geklärt werden muss dann die Kostenübernahme zwischen den Kommunen). Allerdings könnte bei einem klaren vorgegebenen Budget die Kommune viel mehr dazu motiviert werden, Regelsysteme zur Prävention zu stärken und damit Leistungsansprüche zu reduzieren. Das dann entstehende freie Geld könnte wiederum zur weiteren Unterstützung der Regelsysteme eingesetzt werden. der gesetzlichen Vorgaben die finanzielle Leistung bereitstellt. Das ist keinem Entscheidungsträger zu verdenken: Angesichts kurzfristiger Wahlzeiträume und der aktuellen Gesetzeslage wäre es geradezu unsinnig, in Gestaltung und Prävention zu investieren. Die dort getätigten Investitionen rechnen sich nämlich nur selten während der Wahlzeit, und da auch kein Gesetz dazu nötigt (es gibt keinen Einzelfallanspruch) kommt kommunale Politik nie unter Druck, in diesem Bereich systematisch zu investieren.

Wenn jedoch ohnehin eine bestimmte Summe für Jugendhilfe bereitgestellt werden muss, über deren Kürzung gar nicht erst diskutiert werden kann, wird Politik damit konfrontiert, vorhandene Einzelfallansprüche mit sinnvoller Prävention zu verbinden. Es wird dann nicht mehr möglich sein, durch trickreiche Maßnahmen Einsparungen bei den Einzelfallleistungen vorzunehmen, denn die Gesamtsumme für Jugendhilfe muss ohnehin erbracht werden. Es geht dann nicht um die Frage, wie man am besten sparen kann, sondern um die Frage, wie man das vorhandene Geld am besten im Sinne der gesetzlichen Vorgaben und dem Wohlergehen der Kinder, Jugendlichen und Fami- 
lien einsetzt. Dazu braucht es natürlich Hinweise und Anregungen (etwa durch eine Anlage zum Gesetz).

\section{5.}

Durch eine Organisationsreform in den Jugendämtern könnte die Zuständigkeit der Sozialen Dienste konzentriert werden auf den Kinderschutz sowie die Einleitung von Hilfen verbunden mit der Überprüfung der Leistungsansprüche und Leistungserbringung. Die gezielte Weiterentwicklung der Unterstützungsangebote, die Kooperation unterschiedlicher Träger sowie die Mobilisierung von Ressourcen im sozialen Raum wäre dann nicht mehr Aufgabe der Sozialen Dienste.

\section{6.}

Außerschulische Unterstützung von Bedingungen für gelingendes Zusammenleben bedarf einer dauerhaft abgesicherten lokalen Infrastruktur, die Rahmenbedingungen, personelle Kontinuität und dauerhafte Unterstützung schafft - egal, unter welcher Überschrift. Die aktuellen gesetzlichen Rahmenbedingungen bieten in dieser Hinsicht nichts Erfreuliches. Sowohl die Quartiermanager als auch die eher auf Selbsthilfe orientierten Anwälte von bürgerschaftlichem Engagement setzen sich seit vielen Jahren für eine systematische Finanzierung von Infrastruktureinrichtungen ein, die im weitesten Sinne tragende Strukturen im Gemeinwesen fördern.

Einzelne Beispiele dafür gibt es schon. Schaut man sich etwa die Projektförderung seitens der Berliner Senatsverwaltung unter dem Titel »Zuschüsse zur Bildung und Förderung von Stadtteilzentren « an, so zeigt sich, dass hier in durchaus vorbildlicher Weise Stadtteilzentren mit einem gemeinwesenorientierten Ansatz gefördert werden, in denen auch Jugendhilfeaktivitäten konzeptionell wie finanzierungslogisch ihren Platz finden. Derlei gebietsbezogene Standorte aufzubauen und $\mathrm{zu}$ stabilisieren sowie darauf bezogene dauerhafte Förderstrukturen zu etablieren, könnte eine gemeinsame Aufgabe der landes- und bundesweit agierenden Protagonisten der jeweiligen Bereiche darstellen. Unter diesem Dach könnten die konzeptionell hetero- genen, sich indes nicht widersprechenden und in der Praxis gar ausgezeichnet ergänzenden Ansätze zusammengeführt und für einander nutzbar gemacht werden. Somit geht es um eine Ergänzung oder Reduzierung individualisierter Geldströme zugunsten von Finanzierungen für eine bessere soziale Infrastruktur.

\section{7.}

$\mathrm{Zu}$ den Umrissen einer weiterführenden Debatte sollten auch Überlegungen über ein »Bildungsgesetz " gehören, das die Systeme Kita, Schule und Jugendhilfe integriert und ein verzahntes und flexibles Arbeiten der Institutionen normiert. Die Zusammenarbeit von Schule und Jugendhilfe muss vor dem Hintergrund des gemeinsamen Zieles, den Jugendlichen den bestmöglichen Abschluss zu vermitteln deutlich und vor allem zeitnah weiterentwickelt werden.

Abgesehen von einzelnen gut entwickelten Projekten entspricht die gegenwärtige Arbeitsweise der Jugendhilfe und der Schule nicht den genannten Anforderungen. In den Jugendämtern sind die Arbeitsweisen, Zuständigkeiten und Entscheidungsgrundlagen bei den Bewilligungen von Maßnahmen sehr unterschiedlich und in starkem Maße abhängig von den einzelnen Fachkräften. Die Bearbeitungszeiträume von der Antragstellung bis zur Bewilligung sind $\mathrm{zu}$ lang. Die Kooperation von Schule und Jugendhilfe hat einen weitgehend reaktiven Charakter im Sinne einer Schadensbegrenzung. So ist beispielsweise die "Schuldistanziertenarbeit ", wie sie an unterschiedlichen Schulen durchgeführt wird, ein reaktives Arbeitsfeld, da sich bestehende schwierige familiäre Lebensverhältnisse sowie gescheiterte Schulbiografien der jeweiligen Schüler bereits verfestigt haben. Schulmüdigkeit, Schulverweigerung und Schulabbruch und deren Folgen kann man nur in den ersten Grundschuljahren im Sinne von Prävention verhindern. In den weiterführenden Schulen ist dies nach Erfahrungen von Lehrern und Schulpädagogen kaum noch möglich. Auch hier müssen neue verbindliche Formen der Zusammenarbeit zwischen Schule, Schulsozialarbeit, den Hilfeanbietern und Stadtteileinrichtungen entwickelt und über Gesetze verbindlich gemacht werden.
Eine Grundvoraussetzung hierfür ist, die zentralistische Steuerung des Systems Schule aufzugeben. Neue Schulträgermodelle (z. B. Stiftungen) können sich dann etablieren, wenn die Finanzierung der Schulen in diesem Kontext über Entgelte oder Bildungsgutscheine sowie sozialstrukturelle persönliche Zuschläge für sozial benachteiligte Kinder geregelt würde. Schulen, die ihre Ressourcen autonom managen können, sind dann in der Lage, sich für den Sozialraum zu öffnen. Es würde eine Schule neuen Typs entstehen, die weniger sozial selektiv, sondern verstärkt sozial integrativ wirken könnte und damit in ihren Kernkompetenzen, nämlich der Wissensvermittlung und der Persönlichkeitsbildung, gestärkt wird. Bildung im weiten Sinne: das wäre die »Prävention« schlechthin. Also kommt es darauf an, alle Institutionen der Bildung (Kindertagesstätte, Hort, Schule, Kinder- und Jugendfreizeiteinrichtungen, Familienbildungseinrichtungen usw.) in die Lage zu versetzen, eng zusammenarbeiten $\mathrm{zu}$ können (und zu müssen) und die Mittel, die etwa für Erziehungshilfen eingesetzt werden müssen (individueller Rechtsanspruch!) dort einzubinden.

Dass das erfolgreich möglich ist, zeigen u. a. zahlreiche Modelle der Integration behinderter Kinder in Regelkitas und Regelschulen sowie die Integration von Tagesgruppen in Regeleinrichtungen. Ganz nebenher werden mit solchen Verfahren auch die Eltern weitaus besser in die Institutionen eingebunden und entwickeln für sie Interesse, mit dem Ergebnis, dass die Erziehungs- und Sozialkompetenz der heute stark verunsicherten Eltern gestärkt wird. So könnten Erwartungs- und Konsumhaltungen abgelöst werden, die beinhalten: für Erziehung, Bildung und vor allem für Erziehungsprobleme sind zuerst staatliche Instanzen zuständig! Tatsächlich haben wir längst so etwas wie einen »Erziehungshilfemarkt «, der - auch finanziell und wirtschaftlich - bedient sein will und viele Arbeitsplätze trägt und viel Kompetenz bindet. Legitim, legal und sozusagen gesetzlich gewollt.

Wir plädieren dafür, diese Arbeitsplätze, diese Kompetenz, dieses Potenzial an Fähigkeiten aus den genannten Gründen mit den so genannten Regeleinrichtungen zu verbinden. Was daraus entsteht: eine klassische Win-win-Situation für alle Beteiligten! 